Published in final edited form as:

Brain Behav Immun. 2020 November ; 90: 272-278. doi:10.1016/j.bbi.2020.08.029.

\title{
Associations between urinary biomarkers of oxidative stress in the third trimester of pregnancy and behavioral outcomes in the child at 4 years of age
}

\author{
Anna-Sophie Rommel ${ }^{1,{ }^{*}}$, Ginger L. Milne ${ }^{2}$, Emily S. Barrett ${ }^{3,4}$, Nicole R. Bush ${ }^{5}$, Ruby \\ Nguyen $^{6}$, Sheela Sathyanarayana ${ }^{7,8}$, Shanna H. Swan ${ }^{9}$, Kelly K. Ferguson ${ }^{10}$ \\ ${ }^{1}$ Department of Psychiatry, Icahn School of Medicine at Mount Sinai, New York, NY, USA \\ ${ }^{2}$ Division of Clinical Pharmacology, Department of Medicine, Vanderbilt University Medical Center, \\ Nashville, TN, USA \\ ${ }^{3}$ Department of Biostatistics and Epidemiology, Environmental and Occupational Health Sciences \\ Institute, Rutgers School of Public Health, Piscataway, NJ, USA \\ ${ }^{4}$ Department of Obstetrics and Gynecology, University of Rochester School of Medicine and \\ Dentistry, Rochester, NY, USA \\ ${ }^{5}$ Department of Psychiatry and Behavioral Sciences and Department of Pediatrics, University of \\ California, San Francisco, CA, USA \\ ${ }^{6}$ Division of Epidemiology and Community Health, School of Public Health, University of \\ Minnesota, Minneapolis, MN, USA \\ ${ }^{7}$ Seattle Children's Research Institute, Seattle, WA, USA \\ ${ }^{8}$ Department of Pediatrics and Department of Environmental and Occupational Health Sciences, \\ University of Washington, Seattle, WA, USA \\ ${ }^{9}$ Department of Environmental Medicine and Public Health, Icahn School of Medicine at Mount \\ Sinai, New York, NY, USA \\ ${ }^{10}$ Epidemiology Branch, National Institute of Environmental Health Sciences, Research Triangle \\ Park, NC, USA.
}

\section{Introduction}

Neurodevelopmental disorders such as autism spectrum disorder (ASD) and attention deficit hyperactivity disorder (ADHD) are complex and debilitating conditions arising early in

\footnotetext{
*Corresponding author: anna.rommel@mssm.edu; 17 East 102nd Street, New York, NY, USA.

Publisher's Disclaimer: This is a PDF file of an unedited manuscript that has been accepted for publication. As a service to our customers we are providing this early version of the manuscript. The manuscript will undergo copyediting, typesetting, and review of the resulting proof before it is published in its final form. Please note that during the production process errors may be discovered which could affect the content, and all legal disclaimers that apply to the journal pertain.

Declaration of Competing Interest

The authors declare that they have no known competing financial interests or personal relationships that could have appeared to influence the work reported in this paper.
} 
development and persisting across the life span (Thapar, Cooper, \& Rutter, 2017). The etiology of these disorders is poorly understood. However, it is hypothesized that neurodevelopmental disorders stem from genetic factors, environmental insults, or a combination of the two, resulting in some form of disruption in brain development (Thapar et al., 2017).

The most rapid development of the brain occurs in utero (Thomason, 2020). A wealth of epidemiological research has, therefore, aimed to understand the risk of prenatal exposure to common environmental stressors for adverse neurodevelopment (Mandy \& Lai, 2016; Thapar, Cooper, Eyre, \& Langley, 2013). A variety of environmental stressors including pollutants, heavy metals, and certain drugs (e.g. tobacco, alcohol, valproate) have been identified as harmful to fetal neurodevelopment (Landrigan, 2010). They may further play causal or contributing roles in the emergence of developmental disabilities (Mandy \& Lai, 2016; Thapar et al., 2013). The impacts of some of these stressors may vary by maternal education due to structural (e.g., poverty, neighborhood deprivation, pollution) and/or individual-level (e.g., health behaviors, genetics) factors (Machlin, McLaughlin, \& Sheridan, 2020; Sharp, Mangalmurti, Hall, Choudhury, \& Shaw, 2019).

The environmental exposures identified as harmful to fetal neurodevelopment (e.g. pollutants, heavy metals, chemical solvents, and certain drugs), are also known to greatly increase reactive oxygen species production (Pizzino et al., 2017). Reactive oxygen species production is usually a by-product of oxygen metabolism (Ozcan \& Ogun, 2015). An imbalance between the production of reactive oxygen species and antioxidant defenses, known as oxidative stress, may lead to cell damage (Betteridge, 2000).

The exchange of oxygen and nutrients between a mother and her fetus is the most important function of the placenta. Alterations in placental oxidative stress result in altered placental function and ultimately altered fetal growth and/or developmental programming (Jansson \& Powell, 2007). Accordingly, oxidative stress has recently been proposed as a putative pathological mechanism that could lead to the impairments commonly observed in neurodevelopmental disorders (Cattane, Richetto, \& Cattaneo, 2018). It is thought that oxidative stress may impact numerous systems that are disrupted in these disorders, such as the glutamatergic, neuroimmune, dopaminergic and antioxidant systems (Steullet et al., 2017). Yet, little is known about the association between intrauterine exposure to oxidative stress and neurodevelopment in the offspring even though the brain may be particularly vulnerable to the effects of oxidative stress due to its high oxygen demand and the abundance of peroxidation-susceptible lipid cells (Kim, Kim, Rhie, \& Yoon, 2015). Congruently, oxidative stress has been found to play an important role in the pathophysiology of neurodegenerative diseases such as Alzheimer's and Parkinson's disease (Kim et al., 2015). Moreover, cross-sectional studies have reported increased levels of oxidative stress in children with ASD (Chauhan \& Chauhan, 2006; James et al., 2006) and ADHD (Sezen et al., 2016).

In the present study, we sought to examine associations between multiple urinary biomarkers of oxidative stress in the third trimester of pregnancy and behavioral development in the children from The Infant Development and the Environment Study (TIDES) pregnancy 
cohort. 8-isoprostane-prostaglandin-F2a (8-iso-PGF2a), the major 8-isoprostaneprostaglandin-F2a metabolite, and prostaglandin-F2a (PGF2a) were included as biomarkers of oxidative stress. 8-iso-PGF2a is widely studied and considered one of the best biomarkers of oxidative stress because because it is relatively stable, including in human pregnancy, unaffected by dietary lipid intake unlike other oxidative stress measures, and readily detectable in urine (Ferguson et al., 2017; Roberts \& Morrow, 2000). The 8-isoPGF2 $a$ metabolite is generated in the lungs rather than the kidneys and may be a particularly sensitive biomarker of oxidative stress throughout the entire body (Dorjgochoo et al., 2012). We further included PGF2 $a$ as an oxidative stress biomarker linked to inflammation mechanisms (Xu et al., 2015). PGF2a can also reflect oxidative stress but is more generally a reliable marker of inflammation (Ricciotti \& FitzGerald, 2011). Here, we examined the associations of these biomarkers with externalizing problems and social impairments in children aged 4 years (mean age: 4 years, 6 months), hypothesizing that children prenatally exposed to higher levels of oxidative stress biomarkers will be more likely to show higher levels of behavioral problems at 4 years of age.

\section{Methods}

\section{Study population}

TIDES is an ongoing prospective cohort study designed to examine prenatal phthalate exposure in relation to infant genital morphology. Pregnant women were recruited between August 2010 and August 2012 at university-based prenatal clinics in San Francisco, CA (University of California, San Francisco, UCSF), Rochester, NY (University of Rochester Medical Center, URMC), Minneapolis, MN (University of Minnesota, UMN) and Seattle, WA (University of Washington/Seattle Children's Hospital, UW/SCH). Any woman over 18 years old who was able to read and write English (or Spanish at the CA center), who was $<13$ weeks pregnant, whose pregnancy was not medically threatened, and who planned to deliver in a study hospital was eligible to participate. Gestational age was determined by a medical professional as part of routine clinical care using all available information, including last menstrual period and ultrasound data. Participants provided urine samples and completed a questionnaire in each trimester. Of the 969 women who gave consent, 739 (76.3\%) agreed to take part in the follow-up in early childhood. Follow-up visits with participants were conducted around age 4-5 years to investigate behavioral outcomes. At follow-up, 512 mother-child pairs had data on the exposure and at least one of the outcome variables. The institutional review board (IRB) at all participating institutions approved TIDES prior to study implementation and all subjects provided signed informed consent before starting any study activities. IRB approval was also obtained at the Icahn School of Medicine at Mount Sinai, which served as the TIDES Coordinating Center after 2011.

\section{Biomarkers of oxidative stress}

Biomarkers of oxidative stress were measured in maternal third trimester (mean $=32.6$ weeks of gestation, range 25.7-41.1 weeks) urine samples at the Vanderbilt Eicosanoid Core Laboratory, as described in detail elsewhere (Rosen et al., 2019). Urinary concentrations of the following compounds were measured using gas chromatography-negative ion chemical ionization-mass spectrometry employing stable isotope dilution: free 8-iso-prostaglandin 
F2a (8-iso-PGF2a); its primary metabolite (2,3-dinor-5,6-dihydro-15-F2T-isoprostane); and PGF2a. Urine samples were analyzed for urinary specific gravity, an indicator of urine dilution, at the time of sample collection using a hand-held refractometer (MacPherson, Arbuckle, \& Fisher, 2018).

\section{The Behavior Assessment System for Children (BASC-2)}

The BASC Parent Rating Scale, which provides a multidimensional approach to evaluating behavior in children (Reynolds, 2010), was completed when the children were aged 4-5 years. Parents rated their child's behavior on a 4-point Likert scale (1=never to 4=almost always) on each of the following subscales: Social Skills, Leadership, Hyperactivity, Aggression, Conduct Problems, Anxiety, Depression, Somatization, Atypicality, Withdrawal, and Attention Problems, yielding the composite scores Externalizing Problems (Hyperactivity, Aggression, Conduct Problems), Internalizing Problems (Anxiety, Depression, Somatization), and the Behavioral Symptoms Index (Atypicality, Withdrawal, and Attention Problems). All scores are T-scores ( $M=50, S D=10)$, which are adjusted for the age of the child at testing. Since there is no total BASC scale, we focused our primary analysis on the BASC externalizing scale because the assessed behaviors (Hyperactivity, Aggression, Conduct Problems) serve as a good proxy for ADHD, one of the earliest mental disorders to be diagnosed and therefore of interest here. Hyperactivity is a core ADHD symptom (American Psychiatric Association, 2012), while aggression and conduct problems are strongly associated with ADHD (Tarver, Daley, \& Sayal, 2014), and predict ADHD symptoms later in development (Kuja-Halkola, Lichtenstein, D’Onofrio, \& Larsson, 2015). Secondary analyses of the associations between oxidative stress and the Internalizing Problems and Behavioral Symptoms Index scales can be found in Appendix A (Table A1).

\section{Social Responsiveness Scale (SRS-2)}

This 65 -item rating scale, measuring the severity of autism spectrum symptoms, was completed by a parent when the child was aged $4-5$ years. The SRS (Constantino, 2013) generates scores for six subscales: Social Awareness, Social Cognition, Social Communication, Social Motivation, Social Communication and Interaction and Restricted Interests and Repetitive Behavior, as well as a total score based on these subscales. Each item is scored on a scale ranging from 1 (not true) to 4 (almost always true). Raw scores are converted to T-scores (with $\mathrm{M}=50$ and $\mathrm{SD}=10$ ), adjusting for the age of the child at testing. For our analysis, we examined the total score because it is designed for ASD screening and reflects the severity of social deficits.

\section{Statistical Analysis}

We used linear regression models to estimate associations between urinary concentrations of 8-iso-PGF2 $a$, its primary metabolite, and PGF2 $a$ with continuous BASC Externalizing Problems score and the SRS total score. All oxidative stress and behavioral variables were log-transformed. Results in tables are presented in the form of percent change in BASC Externalizing Problems/SRS total scores with an interquartile range (IQR) increase in urinary levels of oxidative stress (see Appendix A, Table A1 for IQRs). To avoid bias and loss of information (Kontopantelis, White, Sperrin, \& Buchan, 2017; Sterne et al., 2009),

Brain Behav Immun. Author manuscript; available in PMC 2021 November 01. 
missing data were imputed based on all available information using additive regression, bootstrapping, and predictive mean matching.

Potential confounders were identified in a literature review. Crude models were adjusted for gestational age at urine sample collection and specific gravity to account for urine dilution. Adjusted models additionally included maternal education, pre-pregnancy body mass index (BMI), race, age, smoking, and alcohol use during pregnancy. The effect modification of education was assessed by including interaction terms between oxidative stress and education levels in an attempt to address the effect modification of unmeasured factors due to structural (e.g., poverty, environmental exposures) or individual-level (e.g., health behaviors) stressors (Hajat, Hsia, \& O'Neill, 2015; Pampel, Krueger, \& Denney, 2010). Women with a high school education or less were coded as 'less educated' ( $\mathrm{n}=60)$, and women with at least some college education or more were coded as 'more educated' $(n=452)$. Post-hoc tests of the effect modification were carried out using the 'emmeans' package in R. All statistical analyses were performed using $\mathrm{R}$ version 3.5.2.

\section{Results}

The analysis included 512 mother-child pairs (for demographic information, see Table 1). Data on gestational age, alcohol use, smoking, and BASC scores were imputed for 32, 33, 24 , and one individual(s) respectively.

In the crude model, total SRS scores at age 4 years showed a significant positive association with third trimester urinary levels of 8 -iso-PGF $2 a(\beta=0.03, p=0.001,95 \% \mathrm{CI}=0.01 ; 0.05)$ and its major metabolite $(\beta=0.05, \mathrm{p}<0.001,95 \% \mathrm{CI}=0.02 ; 0.08)$ (Table 2). BASC-2 Externalizing Problems scores at age 4 years were positively associated with third trimester urinary concentrations of PGF2a $(\beta=0.02, p=0.04,95 \% \mathrm{CI}=0.001 ; 0.04)$ (Table 2$)$.

In the adjusted model, total SRS scores at age 4 years showed a significant positive association with third trimester urinary levels of 8 -iso-PGF $2 a$ ( $\beta=0.02, p=0.04$, $95 \% \mathrm{CI}=0.001 ; 0.04)$. The strength of the association was modified by level of education $\left(\mathrm{p}_{\text {effect modification }}=0.03\right.$ ). Post-hoc tests suggest that the association between total SRS scores at age 4 years and third trimester urinary levels of 8-iso-PGF2a was only significant in more educated mothers $(\beta=0.02,95 \% \mathrm{CI}=0.001 ; 0.04)$ but not in less educated mothers $(\beta=-0.03,95 \% \mathrm{CI}=-0.07,0.02)$. After adjusting for confounding, BASC-2 Externalizing Problems scores at age 4 years were positively associated with third trimester urinary concentrations of PGF2a ( $\beta=0.02, p=0.01,95 \% \mathrm{CI}=0.01 ; 0.04)$ (Table 2$)$. The strength of the association was modified by level of education ( $p_{\text {effect modification }}=0.01$ ). Post-hoc tests suggest that the association between BASC-2 Externalizing Problems scores at age 4 years and third trimester urinary levels of PGF2 $a$ was only significant in more educated mothers $(\beta=0.02,95 \% \mathrm{CI}=0.005 ; 0.04)$ but not in less educated mothers $(\beta=-0.05,95 \% \mathrm{CI}=-0.11$, $0.005)$. A correlation matrix of the outcome, predictor and confounding variables can be found in the appendix (Appendix B, Table B1).

Results for the other two BASC-2 scales (Internalizing and Behavioral Symptoms Index) can be found in the appendix (Appendix C, Table C1). The Behavioral Symptoms Index was 
positively associated with third trimester urinary concentrations of 8-iso-PGF2 $a$ and PGF2a. The Internalizing Problems scale was not significantly associated with third trimester urinary levels of oxidative stress. We further investigated whether nutritional supplements confounded the association (Appendix D). However, adding nutritional supplements to the model did not alter the results (Appendix D, Table D1).

\section{Discussion}

Our results suggest that third trimester oxidative stress, as measured by urinary concentrations of 8-iso-PGF2a, is associated with social impairments at age 4 years. Inflammation in the third trimester of pregnancy, as measured by urinary concentrations of PGF2a, is furthermore associated with behavioral problems at age 4 years. Moreover, our findings indicate that associations of maternal oxidative stress and inflammation with child behavioral outcomes are evident only among mothers with higher education levels.

The positive associations of prenatal 8-iso- $\mathrm{PGF}_{2 a}$ with childhood ASD symptoms, which we identified in this study, are consistent with previous research reporting cross-sectional associations between increased levels of oxidative stress in individuals with ASD (Chauhan \& Chauhan, 2006; James et al., 2006; Osredkar et al., 2019). Our and previous findings, therefore, suggest that oxidative stress may contribute to the development of ASD. Levels of oxidative stress may be generated in response to internal (Harwell, 2007; James et al., 2006; Rossignol \& Frye, 2012) or external (e.g., environmental pollution, metabolism of xenobiotics) sources, which have previously been associated with an increased risk for ASD (Mandy \& Lai, 2016). Future studies will need to investigate whether oxidative stress is the mechanism underlying the associations between environmental stressors and increased risk for ASD.

Immune dysregulation and inflammation have been implicated in several psychiatric disorders, including schizophrenia (Doorduin et al., 2009), bipolar disorder (Rao, Harry, Rapoport, \& Kim, 2010), and depression (Dobos, Korf, Luiten, \& Eisel, 2010). Here, we found that urinary concentrations of an oxidative stress biomarker linked to inflammation mechanisms, PGF2a, were positively associated with behavioral problems, indexing ADHD symptoms, at age 4 years. These findings are in line with an increasing amount of evidence supporting the role of inflammation in the pathophysiology of ADHD (Dunn, Nigg, \& Sullivan, 2019). Several early risk factors that have been proposed for ADHD, including prenatal exposure to maternal infection, pollutants, alcohol, tobacco and maternal obesity, all share an increased maternal inflammatory profile, raising the possibility that inflammation during prenatal development may play a contributing role in the pathophysiology of ADHD (Costenbader \& Karlson, 2006; Shankar et al., 2011; Terasaki \& Schwarz, 2016).

In our study, associations between prenatal exposure to oxidative stress and child behavioral problems were modified by maternal level of education. These effect modifications may be attributable to structural (e.g., poverty, neighborhood deprivation, pollution) and/or individual-level (e.g., health behaviors, genetics) stressors, which are typically inversely associated with educational attainment (Oates \& Schechter, 2016; Stormacq, Van den Broucke, \& Wosinski, 2019). Thus, our results could indicate that structural and individual- 
level stressors may be more important for child behavioral outcomes in the children born to less educated mothers, and are thus dwarfing the more nuanced differences in outcomes that would be attributable to oxidative stress (Machlin et al., 2020; Sharp et al., 2019).

Our findings must be interpreted in light of a number of limitations. Firstly, no clinical assessments were carried out to establish childhood behavioral problems. However, the SRS (Constantino, 2013) and BASC (Jarratt, Riccio, \& Siekierski, 2005; Ostrander, Weinfurt, Yarnold, \& August, 1998; Reynolds, 2010) have good temporal stability, reliability, as well as discriminate and concurrent validity. The parent-rated SRS exhibits substantial agreement with the Autism Diagnostic Interview-Revised (ADI-R), a widely recognized gold standard for establishing a research diagnosis of ASD (Constantino et al., 2003), and the parent-rated BASC exhibits substantial agreement with the Child Behavior Checklist (Doyle, Ostrander, Skare, Crosby, \& August, 1997). Secondly, biomarkers of oxidative stress were measured in a single urine specimen collected in the third trimester of pregnancy. While urinary 8-isoPGF2 $a$ has been shown to be relatively stable throughout pregnancy (Ferguson et al., 2017), our findings may not be generalizable to other timepoints during pregnancy. Lastly, there was loss to follow-up and our sample of less educated mothers was small. The findings, therefore, requiring replication in a large, diverse sample, with urine specimens collected at multiple timepoints during pregnancy.

Despite these limitations, this study has many strengths. The oxidative stress measure 8-isoPGF2 $a$ is one of the best biomarkers of oxidative stress, including during human pregnancy (Ferguson et al., 2017; Roberts \& Morrow, 2000). 8-iso-PGF2a concentration is not significantly affected by differences in dietary lipid intake of participants (Gopaul, Halliwell, \& Änggård, 2000; Richelle et al., 1999). Moreover, the use of 8-iso-PGF2a is preferred over other biomarkers of oxidative stress such as malondialdehyde (MDA) or 8-

hydroxydeoxyguanosine (8-OHdG). MDA is produced from reactions other than lipid peroxidation and has low stability due to rapid enzyme degradation and its high tendency to react with other proteins (Khoubnasabjafari, Ansarin, \& Jouyban, 2015). Quantification of 8-OHdG can differ substantially based on analytic method, and significant variability due to factors such as urine composition and sampling timepoint have been noted (Barregard et al., 2013). In contrast, F2-isoprostanes are chemically stable over time, specific to peroxidation, and reliable (Montuschi, Barnes, \& Roberts, 2004). Lastly, measuring concentrations in urine rather than in plasma allowed us to avoid autoxidation during storage, which can be a concern with plasma measurements (Morrow et al., 1990).

\section{Conclusion}

In the first study to investigate the association between prenatal exposure to oxidative stress and longer-term childhood behavioral problems, we observed that levels of urinary biomarkers of oxidative stress in the third trimester of pregnancy were associated with behavioral problems in the child at age 4 . Specifically, prenatal exposure to oxidative stress (8-iso-PGF2a) was associated with greater social impairments, indexing ASD symptoms, at 4 years of age. Moreover, prenatal exposure to inflammation (PGF2a) was associated with greater behavioral problems, indexing ADHD symptoms, at 4 years of age. These associations were modified by maternal education. In less educated mothers, these

Brain Behav Immun. Author manuscript; available in PMC 2021 November 01. 
associations were not present, while in more educated mothers these associations persisted.

Future research on this topic should examine factors that could explain the lack of

association in the less educated mothers, and investigate the mechanisms underlying the associations in more educated mothers.

\section{Acknowledgements}

This research was supported in part by the Intramural Research Program of the National Institute of Environmental Health Sciences (NIEHS), National Institutes of Health (NIH) (ZIAES103313) and by the following NIEHS, NIH, grants: R01ES016863, R01ES025169, P30ES023515, R01ES016863-02S4, and P30 ES005022.

\section{Appendix A.: Interquartile Ranges (IQR)}

Table A1.

Distributions of oxidative stress biomarkers measured in the third trimester of pregnancy

\begin{tabular}{l|c|c|c|c|c|c}
\hline Biomarker & Minimum & $\mathbf{2 5}^{\text {th }}$ Percentile & Mean & $\mathbf{7 5}^{\text {th }}$ Percentile & Maximum & IQR \\
\hline 8-iso-PGF2a & 0.01 & 0.41 & 1.15 & 1.52 & 6.60 & 1.11 \\
\hline $\begin{array}{l}\text { 2,3-dinor-5,6-dihydro-15-F } \boldsymbol{T}_{\text {- }} \\
\text { isoprostane }\end{array}$ & 0.11 & 0.32 & 0.71 & 0.94 & 6.05 & 0.62 \\
\hline PGF2a & 0.05 & 0.99 & 2.63 & 3.47 & 15.62 & 2.48 \\
\hline
\end{tabular}

$\mathrm{IQR}=$ Interquartile Range 


\section{Appendix B.: Correlation table}

Table B1.

Correlation matrix of the outcome, predictor and confounding variables.

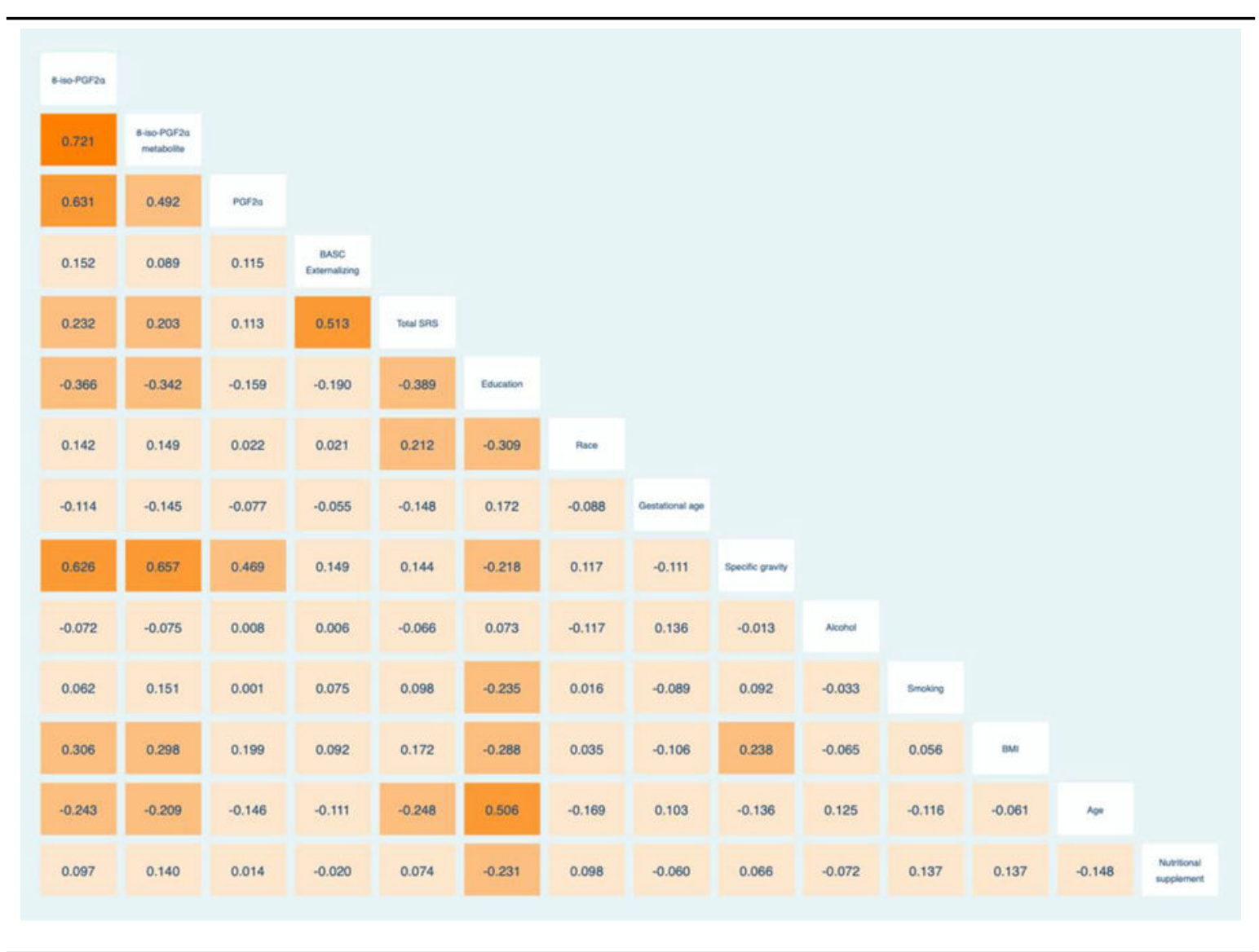

NB: Higher education is coded as having higher numerical values. White ethnicity is coded as having lower numerical values while Non-white ethnicity is coded as having higher numerical values

\section{Appendix C.: Analysis of other BASC subscales}

Table C1.

Crude, adjusted and stratified percent difference (95\% CIs) in BASC internalizing problems and Behavioral Symptom Index (BSI) scores in association with IQR increase in third trimester oxidative stress biomarker levels.

\begin{tabular}{|c|c|c|c|}
\hline & & Crude model $(95 \% \text { CI })^{\dagger}$ & Adjusted model (95\% CI) \\
\hline \multirow{4}{*}{ 8-iso-PGF $F_{2 a}$} & BASC & -0.26 & -0.42 \\
\hline & Internalizing & $(-3.34 ; 3.05)$ & $(-3.82 ; 3.11)$ \\
\hline & \multirow{2}{*}{ BASC BSI } & $3.32^{* * *}$ & $3.23^{*}$ \\
\hline & & $(1.58 ; 6.61)$ & $(0.02 ; 6.55)$ \\
\hline $\begin{array}{l}\text { 2,3-dinor-5,6-dihydro-15-F } F_{2} T- \\
\text { isoprostane }\end{array}$ & BASC & -1.07 & -1.46 \\
\hline
\end{tabular}




\begin{tabular}{|c|c|c|c|}
\hline & & Crude model $(95 \% \text { CI })^{\dagger}$ & Adjusted model (95\% CI) \\
\hline & Internalizing & $(-4.82 ; 2.72)$ & $(-5.56 ; 2.83)$ \\
\hline & \multirow{2}{*}{ BASC BSI } & 1.51 & 0.92 \\
\hline & & $(-2.02 ; 5.17)$ & $(-2.92 ; 4.91)$ \\
\hline \multirow{4}{*}{ PGF2a } & BASC & 0.88 & 1.10 \\
\hline & Internalizing & $(-1.74 ; 3.70)$ & $(-1.73 ; 4.00)$ \\
\hline & \multirow{2}{*}{ BASC BSI } & $2.67^{*}$ & $3.27^{*}$ \\
\hline & & $(0.13 ; 5.28)$ & $(0.65 ; 5.95)$ \\
\hline
\end{tabular}

Signif. Codes:

“****,

0.001

'***, 0.01

'* 0.05 ;

${ }^{\dagger}$ Adjusted for gestational age and specific gravity;

${ }^{*}$ Adjusted for gestational age, specific gravity, maternal education, pre-pregnancy BMI, race, maternal age, smoking and alcohol use during pregnancy; $\mathrm{CI}=$ confidence interval; BASC=Behavior Assessment System for Children; BSI=Behavioral Symptom Index, BMI=body mass index

\section{Appendix D.: Analysis of the covariate nutrional supplements}

\section{Table D1.}

Percent difference (95\% CIs) in BASC externalizing problems and total SRS scores in association with IQR increase in third trimester oxidative stress biomarker levels, additionally adjusting for nutritional supplements.

\begin{tabular}{|c|c|c|}
\hline \multirow{4}{*}{8 -iso-PGF $2 a$} & BASC & 4.26 \\
\hline & Externalizing & $(-8.00 ; 11.76)$ \\
\hline & \multirow{2}{*}{ Total SRS score } & $5.71 *$ \\
\hline & & $(0.28 ; 11.76)$ \\
\hline \multirow{4}{*}{ 2,3-dinor-5,6-dihydro-15-F $F_{2}$-isoprostane } & BASC & 1.48 \\
\hline & Externalizing & $(-8.44 ; 12.48)$ \\
\hline & \multirow{2}{*}{ Total SRS score } & 8.58 \\
\hline & & $(-0.03 ; 18.24)$ \\
\hline \multirow{4}{*}{ PGF2a } & BASC & $3.06 *$ \\
\hline & Externalizing & $(0.63 ; 5.54)$ \\
\hline & \multirow{2}{*}{ Total SRS score } & 1.42 \\
\hline & & $(-0.61 ; 3.50)$ \\
\hline
\end{tabular}

\section{References}

American Psychiatric Association. (2012). Proposed Revision - Attention Deficit/Hyperactivity Disorder. Retrieved January 30, 2012, from http://www.dsm5.org/ProposedRevision/Pages/ proposedrevision. aspx ?rid $=383$

Barregard L, Møller P, Henriksen T, Mistry V, Koppen G, Rossner P, ... Cooke MS (2013). Human and methodological sources of variability in the measurement of urinary 8-oxo-7,8-dihydro- $2^{\prime}$ - 
deoxyguanosine. Antioxidants and Redox Signaling, 18(18), 2377-2391. 10.1089/ars.2012.4714 [PubMed: 23198723]

Betteridge DJ (2000). What is oxidative stress? Metabolism, 49(2), 3-8. 10.1016/ S0026-0495(00)80077-3

Cattane N, Richetto J, \& Cattaneo A (2018). Prenatal exposure to environmental insults and enhanced risk of developing Schizophrenia and Autism Spectrum Disorder: focus on biological pathways and epigenetic mechanisms. Neuroscience and Biobehavioral Reviews. 10.1016/j.neubiorev.2018.07.001

Chauhan A, \& Chauhan V $(2006,8)$. Oxidative stress in autism. Pathophysiology. 10.1016/ j.pathophys.2006.05.007

Constantino JN (2013). Social Responsiveness Scale In Encyclopedia of Autism Spectrum Disorders (pp. 2919-2929). Springer New York 10.1007/978-1-4419-1698-3_296

Constantino JN, Davis SA, Todd RD, Schindler MK, Gross MM, Brophy SL, ... Reich W (2003). Validation of a brief quantitative measure of autistic traits: Comparison of the social responsiveness scale with the Autism Diagnostic Interview-Revised. Journal of Autism and Developmental Disorders, 33(4), 427-433. 10.1023/A:1025014929212 [PubMed: 12959421]

Costenbader KH, \& Karlson EW (2006). Cigarette smoking and autoimmune disease what can we. Lupus, 15(11), 737-745. 10.1177/0961203306069344 [PubMed: 17153844]

Dobos N, Korf J, Luiten PGM, \& Eisel ULM (2010, 3 15). Neuroinflammation in Alzheimer's Disease and Major Depression. Biological Psychiatry. Elsevier USA. 10.1016/j.biopsych.2010.01.023

Doorduin J, De Vries EFJ, Willemsen ATM, De Groot JC, Dierckx RA, \& Klein HC (2009). Neuroinflammation in schizophrenia-related psychosis: A PET study. Journal of Nuclear Medicine, 50(11), 1801-1807. 10.2967/jnumed.109.066647 [PubMed: 19837763]

Dorjgochoo T, Gao Y-T, Chow W-H, Shu X, Yang G, Cai Q, ... Dai Q (2012). Major metabolite of F2isoprostane in urine may be a more sensitive biomarker of oxidative stress than isoprostane itself. The American Journal of Clinical Nutrition, 96(2), 405-414. 10.3945/ajcn.112.034918 [PubMed: 22760572]

Doyle A, Ostrander R, Skare S, Crosby RD, \& August GJ (1997). Convergent and criterion-related validity of the Behavior Assessment System for Children-Parent Rating Scale. Journal of Clinical Child Psychology, 26(3), 276-284. 10.1207/s15374424jccp2603_6 [PubMed: 9292385]

Dunn GA, Nigg JT, \& Sullivan EL (2019, 7 1). Neuroinflammation as a risk factor for attention deficit hyperactivity disorder. Pharmacology Biochemistry and Behavior. Elsevier Inc. 10.1016/ j.pbb.2019.05.005

Ferguson KK, Chen Y-H, VanderWeele TJ, McElrath TF, Meeker JD, \& Mukherjee B (2017). Mediation of the Relationship between Maternal Phthalate Exposure and Preterm Birth by Oxidative Stress with Repeated Measurements across Pregnancy. Environmental Health Perspectives, 125(3), 488-494. 10.1289/EHP282 [PubMed: 27352406]

Gopaul NK, Halliwell B, \& Änggård EE (2000). Measurement of plasma F2-isoprostanes as an index of lipid peroxidation does not appear to be confounded by diet. Free Radical Research, 33(2), 115127. 10.1080/10715760000300671 [PubMed: 10885619]

Hajat A, Hsia C, \& O'Neill MS (2015, 12 1). Socioeconomic Disparities and Air Pollution Exposure: a Global Review. Current Environmental Health Reports. NIH Public Access. 10.1007/ s40572-015-0069-5

Harwell B (2007). Biochemistry of oxidative stress In Biochemical Society Transactions (Vol. 35, pp. 1147-1150). Portland Press 10.1042/BST0351147 [PubMed: 17956298]

James SJ, Melnyk S, Jernigan S, Cleves MA, Halsted CH, Wong DH, ... Gaylor DW (2006). Metabolic endophenotype and related genotypes are associated with oxidative stress in children with autism. American Journal of Medical Genetics, Part B: Neuropsychiatric Genetics, 141(8), 947-956. 10.1002/ajmg.b.30366

Jansson T, \& Powell TL (2007). Role of the placenta in fetal programming: underlying mechanisms and potential interventional approaches. Clinical Science, 113(1), 1-13. 10.1042/CS20060339 [PubMed: 17536998]

Jarratt KP, Riccio CA, \& Siekierski BM (2005). Assessment of attention deficit hyperactivity disorder (ADHD) using the BASC and BRIEF. Applied Neuropsychology, 12(2), 83-93. 10.1207/ s15324826an1202_4 [PubMed: 16083397]

Brain Behav Immun. Author manuscript; available in PMC 2021 November 01. 
Khoubnasabjafari M, Ansarin K, \& Jouyban A (2015). Reliability of malondialdehyde as a biomarker of oxidative stress in psychological disorders. BioImpacts, 5(3), 123-127. 10.15171/bi.2015.20 [PubMed: 26457249]

Kim GH, Kim JE, Rhie SJ, \& Yoon S (2015). The Role of Oxidative Stress in Neurodegenerative Diseases. Experimental Neurobiology, 24(4), 325-340. 10.5607/en.2015.24.4.325 [PubMed: 26713080]

Kontopantelis E, White IR, Sperrin M, \& Buchan I (2017). Outcome-sensitive multiple imputation: A simulation study. BMC Medical Research Methodology, 17(1), 2 10.1186/s12874-016-0281-5 [PubMed: 28068910]

Kuja-Halkola R, Lichtenstein P, D'Onofrio BM, \& Larsson H (2015). Codevelopment of ADHD and externalizing behavior from childhood to adulthood. Journal of Child Psychology and Psychiatry, 56(6), 640-647. 10.1111/jcpp.12340 [PubMed: 25303006]

Landrigan PJ (2010, 4). What causes autism? Exploring the environmental contribution. Current Opinion in Pediatrics. 10.1097/MOP.0b013e328336eb9a

Machlin L, McLaughlin KA, \& Sheridan MA (2020). Brain structure mediates the association between socioeconomic status and attention-deficit/hyperactivity disorder. Developmental Science, 23(1), e12844 10.1111/desc.12844 [PubMed: 31056844]

MacPherson S, Arbuckle TE, \& Fisher M (2018). Adjusting urinary chemical biomarkers for hydration status during pregnancy. Journal of Exposure Science and Environmental Epidemiology, 28(5), 481-493. 10.1038/s41370-018-0043-z [PubMed: 29880833]

Mandy W, \& Lai MC (2016, 3 1). Annual Research Review: The role of the environment in the developmental psychopathology of autism spectrum condition. Journal of Child Psychology and Psychiatry and Allied Disciplines. Blackwell Publishing Ltd. 10.1111/jcpp.12501

Montuschi P, Barnes PJ, \& Roberts LJ (2004). Isoprostanes: markers and mediators of oxidative stress. The FASEB Journal, 18(15), 1791-1800. 10.1096/fj.04-2330rev [PubMed: 15576482]

Morrow JD, Hill KE, Burk RF, Nammour TM, Badr KF, \& Roberts LJ (1990). A series of prostaglandin F2-like compounds are produced in vivo in humans by a non-cyclooxygenase, free radical-catalyzed mechanism. Proceedings of the National Academy of Sciences of the United States of America, 87(23), 9383-9387. 10.1073/pnas.87.23.9383 [PubMed: 2123555]

Oates GR, \& Schechter MS (2016, 9 1). Socioeconomic status and health outcomes: cystic fibrosis as a model. Expert Review of Respiratory Medicine. Taylor and Francis Ltd. 10.1080/17476348.2016.1196140

Osredkar J, Gosar D, Maček J, Kumer K, Fabjan T, Finderle P, ... Vrhovšek MJ (2019). Urinary markers of oxidative stress in children with autism spectrum disorder (ASD). Antioxidants, 8(6). 10.3390/antiox 8060187

Ostrander R, Weinfurt KP, Yarnold PR, \& August GJ (1998). Diagnosing attention deficit disorders with the behavioral assessment system for children and the child behavior checklist: Test and construct validity analyses using optimal discriminant classification trees. Journal of Consulting and Clinical Psychology, 66(4), 660-672. 10.1037/0022-006X.66.4.660 [PubMed: 9735584]

Ozcan A, \& Ogun M (2015). Biochemistry of Reactive Oxygen and Nitrogen Species. 10.5772/61193

Pampel FC, Krueger PM, \& Denney JT (2010). Socioeconomic Disparities in Health Behaviors. Annual Review of Sociology, 36(1), 349-370. 10.1146/annurev.soc.012809.102529

Pizzino G, Irrera N, Cucinotta M, Pallio G, Mannino F, Arcoraci V, ... Bitto A (2017). Oxidative Stress: Harms and Benefits for Human Health. 10.1155/2017/8416763

Rao JS, Harry GJ, Rapoport SI, \& Kim HW (2010). Increased excitotoxicity and neuroinflammatory markers in postmortem frontal cortex from bipolar disorder patients. Molecular Psychiatry, 15(4), 384-392. 10.1038/mp.2009.47 [PubMed: 19488045]

Reynolds CR (2010). Behavior Assessment System for Children In The Corsini Encyclopedia of Psychology (pp. 1-2). Hoboken, NJ, USA: John Wiley \& Sons, Inc. 10.1002/9780470479216.corpsy0114

Ricciotti E, \& FitzGerald GA (2011). Prostaglandins and inflammation. Arteriosclerosis, Thrombosis, and Vascular Biology, 31(5), 986-1000. 10.1161/ATVBAHA.110.207449 
Richelle M, Turini ME, Guidoux R, Tavazzi I, Métairon S, \& Fay LB (1999). Urinary isoprostane excretion is not confounded by the lipid content of the diet. FEBS Letters, 459(2), 259-262. 10.1016/S0014-5793(99)01259-4 [PubMed: 10518031]

Roberts LJ, \& Morrow JD (2000). Measurement of F2-isoprostanes as an index of oxidative stress in vivo In Free Radical Biology and Medicine (Vol. 28, pp. 505-513). Pergamon 10.1016/ S0891-5849(99)00264-6 [PubMed: 10719231]

Rosen EM, van 't Erve TJ, Boss J, Sathyanarayana S, Barrett ES, Nguyen RHN, ... Ferguson KK (2019). Urinary oxidative stress biomarkers and accelerated time to spontaneous delivery. Free Radical Biology and Medicine, 130, 419-425. 10.1016/j.freeradbiomed.2018.11.011 [PubMed: 30445128]

Rossignol DA, \& Frye RE $(2012,4)$. A review of research trends in physiological abnormalities in autism spectrum disorders: Immune dysregulation, inflammation, oxidative stress, mitochondrial dysfunction and environmental toxicant exposures. Molecular Psychiatry. Nature Publishing Group. 10.1038/mp.2011.165

Sezen H, Kandemir H, Savik E, Basmacı Kandemir S, Kilicaslan F, Bilinc H, \& Aksoy N (2016). Increased oxidative stress in children with attention deficit hyperactivity disorder. Redox Report, 21(6), 248-253. 10.1080/13510002.2015.1116729 [PubMed: 26886057]

Shankar K, Zhong Y, Kang P, Lau F, Blackburn ML, Chen J-R, ... Badger TM (2011). Maternal Obesity Promotes a Proinflammatory Signature in Rat Uterus and Blastocyst. Endocrinology, 152(11), 4158-4170. 10.1210/en.2010-1078 [PubMed: 21862610]

Sharp W, Mangalmurti A, Hall C, Choudhury S, \& Shaw P (2019). Associations between neighborhood, family factors and symptom change in childhood attention deficit hyperactivity disorder. Social Science and Medicine. 10.1016/j.socscimed.2019.02.054

Sterne JAC, White IR, Carlin JB, Spratt M, Royston P, Kenward MG, ... Carpenter JR (2009, 7 18). Multiple imputation for missing data in epidemiological and clinical research: Potential and pitfalls. BMJ (Online). British Medical Journal Publishing Group. 10.1136/bmj.b2393

Steullet P, Cabungcal JH, Coyle J, Didriksen M, Gill K, Grace AA, ... Do KQ (2017, 7 1). Oxidative stress-driven parvalbumin interneuron impairment as a common mechanism in models of schizophrenia. Molecular Psychiatry. Nature Publishing Group. 10.1038/mp.2017.47

Stormacq C, Van den Broucke S, \& Wosinski J (2019). Does health literacy mediate the relationship between socioeconomic status and health disparities? Integrative review. Health Promot Int, 34(5), e1-e17. [PubMed: 30107564]

Tarver J, Daley D, \& Sayal K (2014). Attention-deficit hyperactivity disorder (ADHD): an updated review of the essential facts. Child: Care, Health and Development, 40(6), 762-774. 10.1111/ cch.12139

Terasaki LS, \& Schwarz JM (2016). Effects of Moderate Prenatal Alcohol Exposure during Early Gestation in Rats on Inflammation across the Maternal-Fetal-Immune Interface and Later-Life Immune Function in the Offspring. Journal of Neuroimmune Pharmacology, 11(4), 680-692. 10.1007/s11481-016-9691-8 [PubMed: 27318824]

Thapar A, Cooper M, Eyre O, \& Langley K (2013). What have we learnt about the causes of ADHD? Journal of Child Psychology and Psychiatry, and Allied Disciplines, 54(1), 3-16. 10.1111/ j.1469-7610.2012.02611.x

Thapar A, Cooper M, \& Rutter M (2017, 4 1). Neurodevelopmental disorders. The Lancet Psychiatry. Elsevier Ltd. 10.1016/S2215-0366(16)30376-5

Thomason ME (2020). Development of Brain Networks in Utero: Relevance for Common Neural Disorders. Biological Psychiatry. 10.1016/j.biopsych.2020.02.007

Xu C, Liu W, You X, Leimert K, Popowycz K, Fang X, ... Ni X (2015). PGF2a modulates the output of chemokines and pro-inflammatory cytokines in myometrial cells from term pregnant women through divergent signaling pathways. Molecular Human Reproduction, 21(7), 603-614. 10.1093/ MOLEHR/GAV018 [PubMed: 25882540] 


\section{Highlights}

Prenatal oxidative stress is linked to greater social impairment at age 4 years

Prenatal inflammation is linked to greater behavioral problems at age 4 years

Maternal education modifies the relationship of oxidative stress with child behavior 
Table 1.

Demographics of the study sample: mean (standard deviation) or \% (n).

\begin{tabular}{|c|c|c|c|c|}
\hline & & Whole Sample $(\mathrm{n}=512)$ & Less Educated $(\mathrm{n}=60)$ & More Educated $(n=452)$ \\
\hline \multicolumn{2}{|c|}{ Maternal age in years } & $32.7(3.4)$ & $24.9(5.5)$ & $32.4(5.5)$ \\
\hline \multicolumn{2}{|c|}{ Maternal pre-pregnancy BMI kg/m² } & $26.0(6.3)$ & $29.4(9.2)$ & $25.5(5.8)$ \\
\hline \multirow{3}{*}{ Race \% } & White & $72.7(372)$ & $23.3(14)$ & $79.2(358)$ \\
\hline & African American & $9.6(49)$ & $46.7(28)$ & $4.6(21)$ \\
\hline & Other & $17.8(91)$ & $30.0(18)$ & $16.2(73)$ \\
\hline \multicolumn{2}{|c|}{ Gestational age at urine sample collection in weeks } & $32.7(3.4)$ & $31.4(3.5)$ & $32.8(3.3)$ \\
\hline \multicolumn{2}{|c|}{ Child sex (\% male) } & $47.7(244)$ & $41.7(25)$ & $48.5(219)$ \\
\hline \multicolumn{2}{|c|}{ Child age in months } & $54.0(4.5)$ & $54.0(4.6)$ & $53.9(3.3)$ \\
\hline \multirow{6}{*}{ Education \% } & 8th grade or lower & $0.2(1)$ & $1.7(1)$ & I \\
\hline & Some high school & $6.1(31)$ & $51.7(31)$ & I \\
\hline & Graduated high school & $5.5(28)$ & $46.7(28)$ & I \\
\hline & $\begin{array}{l}\text { Some college or technical school (not } \\
\text { graduated) }\end{array}$ & $9.8(50)$ & I & $11.1(50)$ \\
\hline & Graduated college or technical school & $30.9(158)$ & I & $35.0(158)$ \\
\hline & Some graduate work or graduate degree & $47.7(244)$ & I & $54.0(244)$ \\
\hline \multirow{2}{*}{ Alcohol use } & Yes & $7.8(40)$ & - & $8.8(40)$ \\
\hline & No & $92.2(472)$ & $100(60)$ & $91.2(412)$ \\
\hline \multirow{2}{*}{ Smoking \% } & Yes & $3.5(18)$ & $16.7(10)$ & $1.8(8)$ \\
\hline & No & $96.5(494)$ & $83.3(50)$ & $98.2(444)$ \\
\hline \multirow{4}{*}{ Center \% } & UCSF & $27.5(141)$ & $5.0(3)$ & $30.5(138)$ \\
\hline & UMN & $28.5(146)$ & $6.7(4)$ & $31.4(142)$ \\
\hline & URMC & $26.2(134)$ & $85.0(51)$ & $18.4(83)$ \\
\hline & UW & $17.8(91)$ & $3.3(2)$ & $19.7(89)$ \\
\hline \multicolumn{2}{|c|}{ BASC Externalizing Problems } & $49.6(9.1)$ & $52.8(13.6)$ & $49.1(8.4)$ \\
\hline \multicolumn{2}{|c|}{ Total SRS score } & $45.1(7.2)$ & $51.7(8.4)$ & $44.2(6.2)$ \\
\hline
\end{tabular}

BASC=Behavior Assessment System for Children; SRS=Social Responsiveness Scale, BMI=body mass index; UCSF=University of California, San Francisco; UMN=University of Minnesota, Minneapolis, MN; URMC= University of Rochester Medical Center, Rochester, NY;

UW=University of Washington/Seattle Children's Hospital, Seattle, WA, Missing data on gestational age, alcohol use, smoking, and BASC scores were imputed for $32,33,24$, and one individual(s) respectively. The other variable did not have missing values. 
Table 2.

Crude and adjusted percent difference (95\% CIs) in BASC externalizing problems and total SRS scores in association with IQR increase in third trimester oxidative stress biomarker levels in the whole sample.

\begin{tabular}{|c|c|c|c|}
\hline & & Crude model $(95 \% \mathrm{CI})^{\dagger}$ & Adjusted model $(95 \% \mathrm{CI})^{*}$ \\
\hline \multirow{4}{*}{ 8-iso-PGF $F_{2 a}$} & BASC & 1.85 & 1.85 \\
\hline & Externalizing & $(-1.04 ; 4.68)$ & $(-1.23 ; 4.94)$ \\
\hline & \multirow{2}{*}{ Total SRS score } & $4.00^{* *}$ & $2.58^{*}$ \\
\hline & & $(1.58 ; 6.61)$ & $(0.08 ; 5.16)$ \\
\hline \multirow{4}{*}{ 2,3-dinor-5,6-dihydro-15- $F_{2}$ T-isoprostane } & BASC & 0.54 & 0.14 \\
\hline & Externalizing & $(-2.75 ; 3.94)$ & $(-3.51 ; 3.93)$ \\
\hline & \multirow{2}{*}{ Total SRS score } & $5.62^{* * * *}$ & 2.95 \\
\hline & & $(2.61 ; 8.73)$ & $(-0.13 ; 6.13)$ \\
\hline \multirow{4}{*}{ PGF2a } & BASC & $2.54 *$ & $3.13 *$ \\
\hline & Externalizing & $(0.13 ; 4.88)$ & $(0.63 ; 5.69)$ \\
\hline & \multirow{2}{*}{ Total SRS score } & 1.77 & 1.42 \\
\hline & & $(-0.38 ; 3.83)$ & $(-0.61 ; 3.50)$ \\
\hline
\end{tabular}

Signif. Codes:

****,

0.001

“***, 0.01

; 3.05 ;

${ }^{\dagger}$ Adjusted for gestational age and specific gravity;

${ }^{*}$ Adjusted for gestational age, specific gravity, maternal education, pre-pregnancy body mass index, race, age, smoking, and alcohol use during pregnancy; $\mathrm{CI}=$ confidence interval; BASC=Behavior Assessment System for Children; SRS=Social Responsiveness Scale. 